\title{
Herausforderungen für den Bildungshausbau
}

\author{
Über die Rolle pädagogischer Vorstellungen beim Bau von \\ Bildungshäusern
}

Monika Schopper*

https://doi.org/10.53349/resource.2021.i16.a984

\section{Zusammenfassung}

Der Bildungshausbau ist Thema aktueller Debatten in der Stadtentwicklung und Stadtplanung sowie in der Pädagogik. Viele Expert*innen beschäftigen sich in Studien mit Fragen zu gutem und gelingendem Schulbau. Die Anforderungen der Gesellschaft an Bildungshäuser verändern sich, wenn in ganztägigen Schulformen nicht nur Unterricht, sondern auch Freizeitbetreuung für die Schüler*innen stattfinden soll. Gleichzeitig soll Schule ein Ort der Begegnung und Kommunikation, des sozialen Lernens und der Kooperation sein. Schule ist in vielfacher Hinsicht in Bewegung. Um mit den Veränderungen und Ansprüchen Schritt zu halten, steht der Bildungshausbau immer wieder vor Herausforderungen. Einerseits werden Leuchtturmprojekte geschaffen, andererseits entstehen nach wie vor Bildungsbauten, die den gegenwärtigen Anforderungen und zukünftigen Entwicklungen nicht gerecht werden (Schopper, 2021).

\section{Abstract}

The construction of educational buildings is the current subject of debate in urban development and planning as well as in pedagogy. Many experts are trying to address the question of what makes a good and functional educational building. Society's demands are changing with regards to educational buildings, particularly for allday schooling, where not only education but also after-school care and recreation have to be taken into account. At the same time, schools should be places of social interaction, communication, social learning and cooperation. In so many ways, schools are constantly in motion. Educational constructions constantly face challenges in order to keep up with the changes and demands. On one hand, landmark projects are being developed, while on the other hand, educational buildings are still being built that fail to meet current requirements or future developments (Schopper, 2021).

\begin{tabular}{ll}
\hline Schlüsselwörter: & Keywords: \\
Pädagogik & Pedagogy \\
Architektur & Architecture \\
Schulhausbau & Construction of educational buildings
\end{tabular}

\section{Einleitung}

Seit vielen Jahren machen Beispiele guter, innovativer Schulbauten Schlagzeilen und werden in Studien als richtungsweisend vorgestellt. Gleichzeitig zeigt die Praxis, dass die Umsetzung solcher gelungener Bildungshäuser keine Selbstverständlichkeit ist und Bauten entstehen, die über ein Mindestmaß an Anforderungen nicht hinauskommen. Für den Schulbau ergibt sich die Schwierigkeit, Pädagogik und Architektur in ein gut funktionierendes harmonisches Ganzes zu übersetzen, um den Anforderungen der Schulentwicklung in Gestaltung, Funktionalität und Praktikabilität von Räumen sowie städtebaulichen Konzepten zu entsprechen. Forschungsergebnisse, Empfehlungen und Erkenntnisse für gute Schulbauten gelangen dabei nur eingeschränkt zur Umsetzung (u. a. Hübner, 2016; Lingg, 2016; Montag Stiftung, 2013; Walden \& Borrelbach, 2014).

\footnotetext{
*Pädagogische Hochschule Niederösterreich, Mühlgasse 67, 2500 Baden. E-Mail: monika.schopper@ph-noe.ac.at
} 
Mit dieser Problemlage beschäftigt sich der vorliegende Artikel. Es wird gezeigt, welche pädagogischen Vorstellungen Architekt*innen, Pädagog*innen und Mitarbeiter*innen von Bildungsbauten, Vertreter*innen der Verwaltung und der Behörde beim Bau von Bildungshäusern leiten und in welchem Zusammenhang deren Deutungsmuster mit den Strukturen des Planungsprozesses stehen. Dabei wird auf Grundlagen und Ergebnisse einer Fallstudie im Zuge eines Promotionsprojektes Bezug genommen (Schopper, 2021). Der Forschungsfokus mit dem Blick auf pädagogische Deutungsmuster und Entwicklungen im Planungsprozess beleuchtet einen bislang nicht untersuchten Aspekt in Studien zum Bildungsbau. Im Zuge der Auswertung wurde ein Modell eines Handlungs-Struktur-Gefüges (in Anlehnung an Giddens, 1988) für den Bildungsbau in Verbindung mit pädagogischen Vorstellungen von Beteiligten entwickelt.

Das Bauen von Bildungshäusern versteht sich als sozialer Prozess, der von Architekturschaffenden fordert, ihre eigenen Vorstellungen und ihre Selbstverwirklichung in den Hintergrund zu rücken und jenen Personen und deren Argumenten Raum zu geben, die in diesen Bauten arbeiten (leben) und sich wohl fühlen müssen (Hübner, 2016, S. 117). Die Partizipation von Beteiligten wird als wichtig und notwendig erachtet und die Bedeutung ist anhand zahlreicher Studien belegt (u. a. Braum \& Hamm, 2010; die Baupiloten, 2016; Berdelmann, Burri, Dinsleder, Johann, Kirchgässner, Laros, Möhring, Schumacher \& Vollmer, 2016; Burgdorff \& Imhäuser, 2012; Gaus-Hegner, 2009; Hofmann, 2013; Jäger-Klein \& Plakolm-Forsthuber, 2012; Linder, 2015; Lingg, 2016; Meuser, 2014; Montag Stiftung, 2012 und 2013; Pampe, 2016; Plattform SchulUMbau, 2010; Schäfers, 2014; Sprecher Mathieu, 2010 und 2013; Watschinger \& Kühebacher, 2007; von Zadow, 2009).

Wissenschaftliche Erkenntnisse zu gutem Schulbau oder der Bedeutung von Partizipation finden kaum Eingang in die Praxis, ein Phänomen, das ganz allgemein aus der Wissenschaftstransferforschung (Beck \& Bonß, 1989) bekannt ist. „Der Transfer von Wissen ist ein elementarer Bestandteil der Tätigkeit von professionellen Wissensarbeitern und er ist nur durch eine kontinuierliche und bislang häufig unbeachtete und unterschätzte Beziehungs- und Strukturarbeit möglich“ (Oestreicher, 2014, S. 336).

\section{Theoretische Grundlagen}

Zur Beantwortung aufgeworfener Fragestellungen sind die theoretischen Grundlagen von Alltagswissen, Deutungsmustern und dem Konzept von Handlung und Struktur (nach Giddens 1984, 1988) von Bedeutung. Für ein besseres Verständnis wird ein kurzer Überblick über Definitionen und Grundlagen gegeben.

Alltagswissen wird als "Grundlage und Bestandteil jedes wissenschaftlichen Wissens" (Arbeitsgruppe Bielefelder Soziologen, 1981, S. 221f) verstanden. In anderen Worten ausgedrückt ist Alltagswissen der „Bestand an täglichem Wissen, auf dessen Grundlage die gesellschaftliche Wirklichkeit erfahren wird“ (Matthes \& Schütze, 1981, S. 16). Bei Schulbauten geht es einerseits um das Verhältnis von Alltagswissen und professionell-architektonischem Wissen und andererseits um das Verhältnis von Alltagswissen und professionell-pädagogischem Wissen.

Deutungsmuster werden als „Tiefenstruktur menschlichen Bewusstseins" (Dewe \& Ferchhoff, 1991, S. 76) verstanden. Nach Arnold handelt es sich um einen „Begriff zur Erfassung subjektiver Vorstrukturen“ (Arnold, 1985, S. 20f). Die Entwicklung des Deutungsmuster-Begriffs und der Deutungsmusteranalysen geht zurück auf ein Manuskript von Oevermann (1973).

Gesellschaftliche Strukturen rahmen architektonisches Handeln (u. a. in Studien von Kemnitz, 2003; Lingg, 2016; Prechter, 2013; Tosch, 2003 ersichtlich). Zur Erfassung des komplexen Zusammenhangs von Handlung und Struktur und Pädagogik und Architektur wurde für die Untersuchung im Zuge der Analyse der Projektstrukturen auf die Strukturtheorie von Giddens $(1984,1988)$ zurückgegriffen. Diese legt unter anderem die "Dualität von Handlung und Struktur" dar und meint damit, dass Handlung und Struktur einander nicht gegenüberstehen, sondern dass Strukturen in Handlungen einfließen und zugleich Handlungen der Akteur*innen Strukturen verändern oder diese schaffen. Strukturen geben Sicherheit bei der Bewältigung des Alltags und ermöglichen unterschiedliche Abläufe und Prozesse, sie erzeugen aber auch Grenzen und schränken das Handeln ein. Kennzeichnend ist ein prozesshafter, rekursiver Charakter, weil Struktur sich immerwährend durch das praktische Handeln der Akteur*innen wandelt (Neuberger, 1995, S. 305). Den Ansatz von Giddens zeichnet der „Fokus auf Dualität und Prozessualität" aus und die „Strukturationstheorie [gilt] heute als eine der prominenteren (Inter-) Organisationstheorien“ (Sydow \& Wirth, 2014, S. 9). 


\section{Empirische Untersuchung im Überblick}

Die Studie begleitet einen Bauprozess von zwei Bildungsbauten von den Anfängen bis zur Fertigstellung in einem Zeitraum von mehr als zehn Jahren. Grundbedingung für die Durchführung der Untersuchung war das Vorliegen von Bauprojekten, die qualitativ dicht erfasst werden können (eine Anforderung, die nur wenige Projekte aufweisen, da Schulbauprozesse über mehrere bzw. viele Jahre laufen und Zugangsmöglichkeiten zu Datenmaterial oft nur eingeschränkt vorhanden sind). Gegenstand der empirischen Untersuchung sind zwei Bau-Fälle, die im Rahmen einer Fallstudie bearbeitet wurden. Die Fallauswahl anhand theoretischer Überlegungen charakterisiert eine theoriegeleitete Stichprobe (zurückgehend auf Glaser und Strauss, 1967, 1999). An Schulbauprozessen sind Personen aus unterschiedlichen Professionsbereichen beteiligt, die im Zuge narrativer Interviews befragt wurden: Architekt*innen, Behördenvertreter*innen, Leitungen der Bildungshäuser, Pädagog*innen und Vertretungen der Verwaltung und des Schulmanagements.

Die Datenauswertung erfolgte auf Grundlage der dokumentarischen Methode (Bohnsack et al., 2013; Nohl, 2017), die angemessen ist, um „überindividuell geltende und logisch konsistent miteinander verknüpfte Sinninterpretationen sozialer Sachverhalte“ (Dewe, 1984) zu erheben. Die dokumentarische Methode zielt darauf $a b$, Orientierungsrahmen zu erfassen, die anhand der Alltagspraxis sichtbar werden, um dem Fremdverstehen einen methodisch kontrollierten Zugang zu ermöglichen (Kleemann, Krähnke \& Matuschek, 2013, S. 160).

Orientierungsrahmen und Deutungsmuster stehen in einem unmittelbaren Zusammenhang und werden als synonym für die Untersuchung verstanden, denn in beiden Fällen geht es um die Erfassung tiefer liegender Sinngehalte. Formulierende Interpretation, reflektierende Interpretation und komparative Analyse münden in einer Typenbildung. Deutungsmuster „haben die Eigenschaft eines ,tacit knowledge' bzw. eines ,impliziten Wissens', was zur Konsequenz hat, dass die Akteure die innere Logik derjenigen Deutungsmuster, die ihre Wahrnehmung von Welt strukturieren und ihrem Handeln eine Orientierung geben, gewöhnlich nicht oder allenfalls bruchstückhaft explizieren können“ (Bohnsack, Geimer \& Meuser, 2018, S. 40) (Schopper, 2021).

Als Befragungsform für die Untersuchung wurde das narrative Interview herangezogen (Lamnek \& Krell, 2016), das sich anbietet, um subjektbezogenes Erfahrungswissen, Deutungsmuster und Einschätzungen von Beteiligten im Bildungsbau zu erheben und den Entstehungsprozess des Projekts erfassen zu können.

Die Studie gliedert sich in zwei Auswertungsschritte, wodurch das Verhältnis von Handlung und Struktur und die Deutungsmuster beteiligter Personen herausgearbeitet werden können: die Analyse der Projektstrukturen und die Analyse der Deutungsmuster. Es werden Projektstrukturen identifiziert und ins Verhältnis zu Deutungsmustern gebracht (zwei Auswertungsbereiche, siehe Abb. 1), um zu zeigen, wie diese Deutungsmuster auf Strukturen zurückgreifen, oder anders ausgedrückt, wie Akteur*innen ihr Handeln auf Strukturen beziehen und auf diese Weise ein Handlungs-Struktur-Geflecht entsteht. Das rekursive Element der Dualität von Giddens (Giddens, 1984, 1988; Neuberger, 1995) kennzeichnet das stetige, wechselseitige Zusammenspiel von Handlung und Struktur, wobei es nicht darum geht, ob die Handlung oder die Struktur zuerst auf das jeweils andere Element Einfluss nimmt (Sydow \& Wirth, 2014, S. 31f). Der formelle Rahmen von Baubesprechungen im Schulhausbau ermöglicht z. B. das Aushandeln unterschiedlicher Interessen und legt Richtlinien für ein weiteres Vorgehen im Bauprozess fest. Gleichzeitig bestimmen Handlungen innerhalb dieser im Ablauf festgelegten Struktur, wie das konkrete Vorgehen im Detail aussieht, welche Vorstellungen sich durchsetzen oder auf welche Weise gemeinsame Lösungen gefunden werden. 


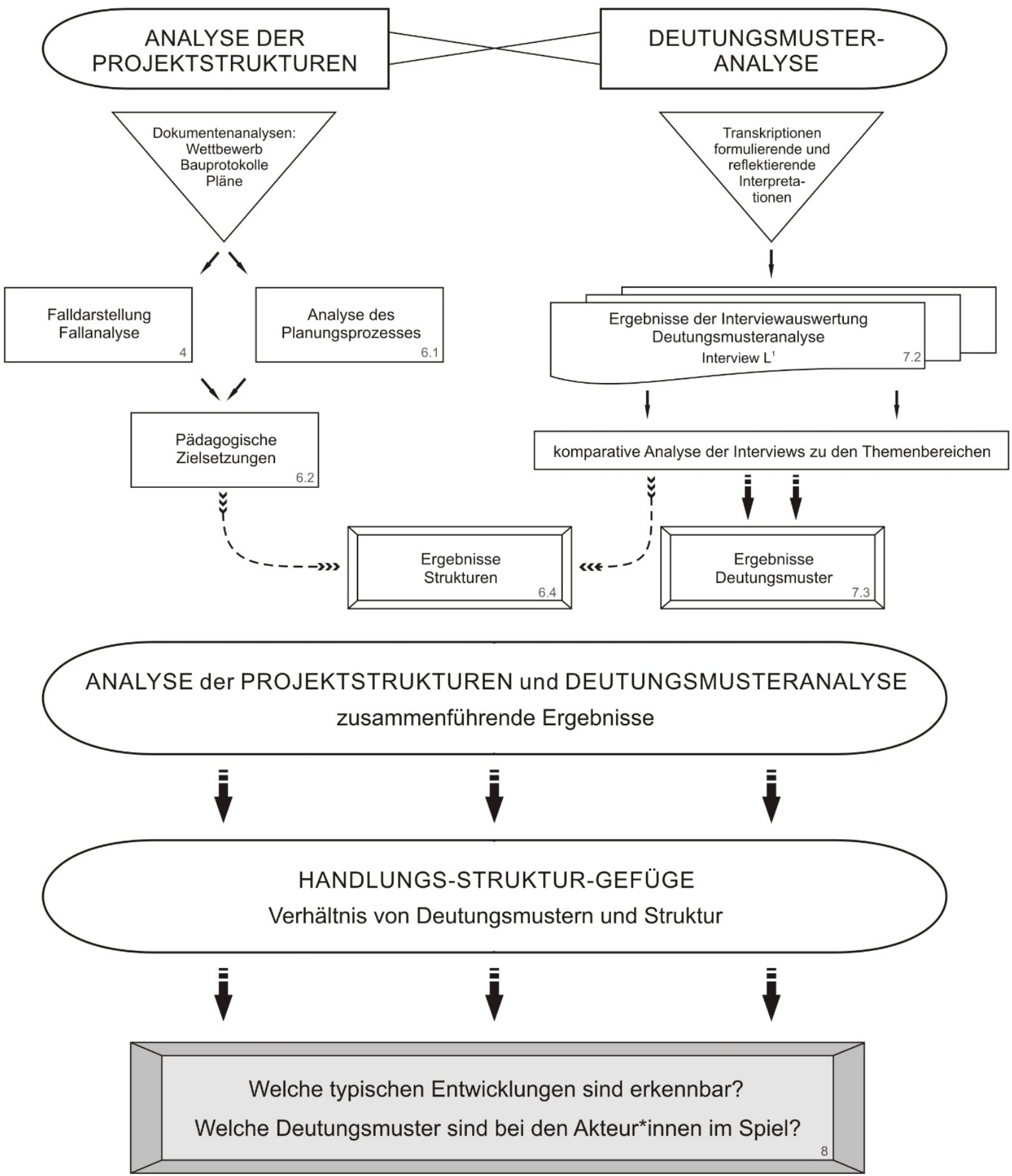

Abbildung 1: Auswertungsschritte (eigene Darstellung)

Die Analyse der Projektstrukturen ergab acht wesentliche Strukturen (siehe Abb. 2): Projektstrukturen, ökonomische Strukturen, baurechtliche Strukturen, räumliche Strukturen, Kooperationsstrukturen, pädagogische Strukturen, Zeitstrukturen und Entscheidungsstrukturen. Die Zusammenführung von Strukturen und Deutungsmustern der Akteur*innen, die diese Strukturen mit Leben füllen, trägt zum Verständnis bei, warum bestimmte Gruppen involvierter Personen nach architektonischen, pädagogischen, finanziellen oder anderen Grundsätzen denken und handeln (Schopper, 2021). 


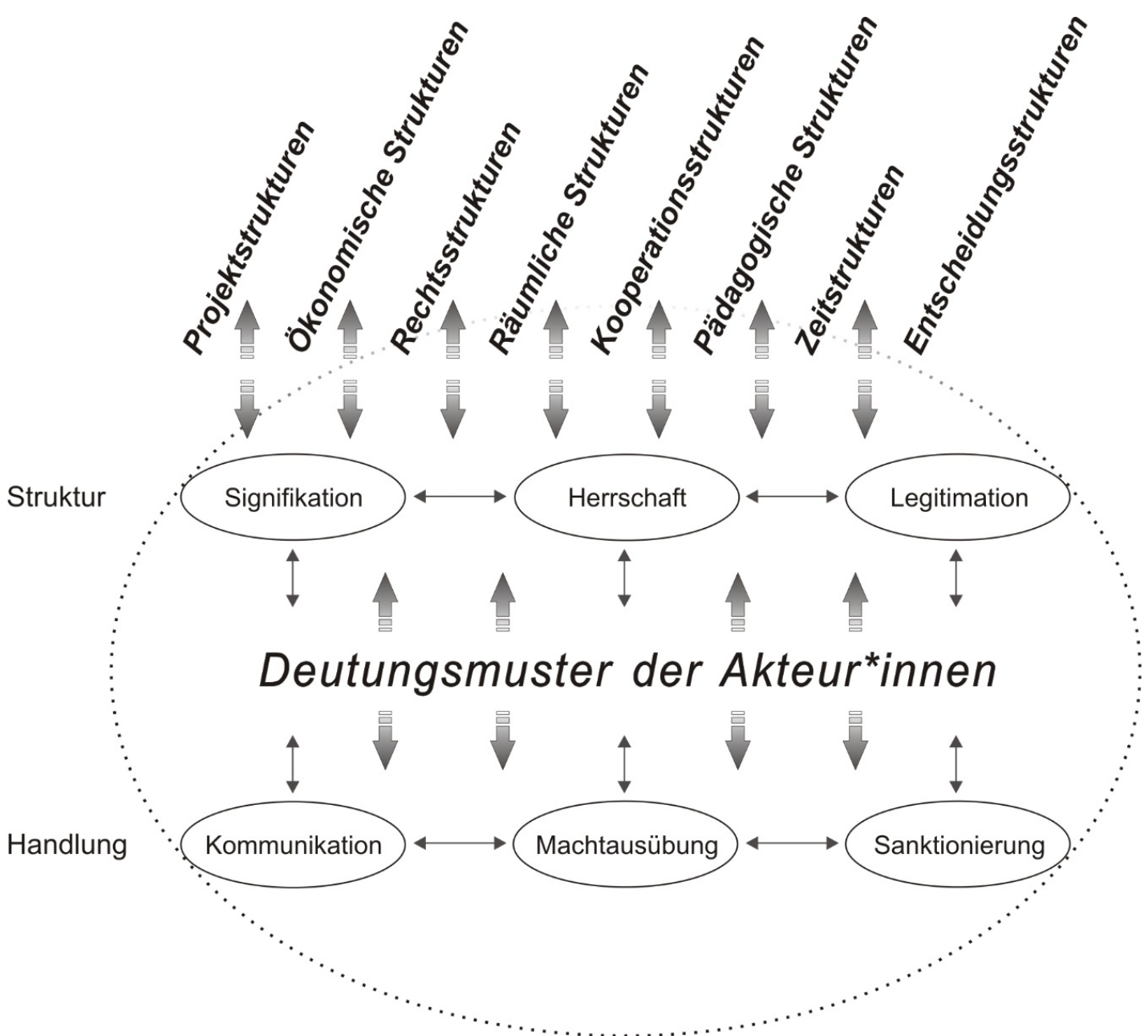

Abbildung 2: Deutungsmuster im Kräftespiel der Strukturen (eigene Darstellung mit Bezug zu Giddens, 1988, S. 81)

Abbildung 2 visualisiert die Strukturen und die sich beeinflussenden Kräfte im Verlauf des Planungsprozesses und der Umsetzung der Bildungsbauten mit Bezugnahme auf das Konzept der Dualität von Struktur (Giddens, 1988). Kommunikation (Strukturdimension der Signifikation bzw. Bedeutung) und Sanktionierung (Strukturdimension der Legitimation) sowie Machtausübung (Strukturdimension der Herrschaft) sind als Bestandteile des Handelns zu sehen (Giddens, 1988). Akteur*innen haben generell die Option zur Ausübung von Macht, wenngleich die Möglichkeiten nicht gleich verteilt sind (ressourcenabhängig). Ökonomische und räumliche Strukturen, Projektstrukturen, Zeitstrukturen oder Entscheidungsstrukturen sind z. B. auf dieser Ebene zu nennen. Wirtschaftliches und technisches Handeln setzen dabei ökonomische und technische Machtmittel ein. Das sind einerseits allokative Ressourcen, z. B. Investitionsbudget, Rohstoffe oder Technik, und andererseits autoritative Ressourcen, wie beispielsweise Arbeitsorganisation, Verwaltung oder Planungsinstrumente (Schwarz, 2017, S. 77f). Bedeutungsstrukturen oder Strukturen der Signifikation nach Giddens Modell von Handlung und Struktur ermöglichen oder behindern kommunikatives Handeln und sind im Zuge der Bauprojekte vor allem bei den Strukturen der Kooperation und Pädagogik relevant. Kommunikation ist nach Giddens (1988, S. 81) über interpretative Schemata (z. B. Wahrnehmungsmuster, Leitbilder) mit der Signifikation verbunden (Schwarz, 2017, S. 78). Regeln und rechtliche Normen ermöglichen oder behindern soziales Handeln (Strukturdimension der Legitimation), Sanktionierungen (auf der Ebene der Handlungen) sind als Bewertungen des Handelns zu verstehen (gut, schlecht, zu teuer, etc.). Dieser Dimension nach Giddens sind die Rechtsstrukturen zuzuordnen. Ebenso können in Teilbereichen Projektstrukturen oder Entscheidungsstrukturen betroffen sein, da die Strukturen der Bauprojekte einander bei bestimmten Themen überlappen (Schopper, 2021).

Anhand der Deutungsmusteranalyse konnten zahlreiche Deutungsmuster analysiert werden. Dabei zeigte sich, dass sie häufig berufsgruppenspezifisch zuordenbar sind, wodurch sich typische Muster für Pädagog*innen, 
Architekt*innen, Behörde und Verwaltung ergaben. Jede der genannten Gruppen kann insgesamt drei Typen der Orientierung im Zuge eines Schulbauprozesses zugeordnet werden: aktive Mitgestalter*innen, Beobachter*innen (unterteilt in abwartende und vertrauende Beobachter*innen), Steuernde (unterteilt in umsetzungsorientiert, gestaltungsorientiert und prozessorientiert Steuernde).

\section{Zentrale Ergebnisse}

Beispielhaft wird nachfolgend auf wesentliche Ergebnisse der Studie eingegangen (Schopper, 2021).

- Akteur*innen transportieren im Rahmen ihrer Projektaufgaben im Bildungsbau zahlreiche Vorstellungen. Dabei kommen pädagogische Deutungsmuster weniger zur Geltung als andere. Für die bearbeiteten Schulbauten können „Moderner Lehrbetrieb“, „Lernende im Mittelpunkt unterrichtlichen Geschehens" und „Lernen im Dialog“ genannt werden.

- Pädagog*innen argumentieren nicht mit wissenschaftlichen Erkenntnissen zu gutem Schulbau, sondern nehmen Bezug auf ihr eigenes pädagogisches Selbstverständnis (siehe Transferproblem). Zum Beispiel berufen sie sich in Planungsgesprächen nicht auf Studien und Normen sogenannter guter Schulbauten.

- Auf der anderen Seite berücksichtigen Architekt*innen aktuelle pädagogische Studien und Vorgaben nur eingeschränkt bei der Projektplanung.

- Im Zuge einer Typenbildung entlang der Berufsgruppen konnten drei Haupttypen von Akteur*innen definiert werden: aktive Mitgestalter*innen, Beobachter*innen und Steuernde.

- Es wurde ein Handlungs-Struktur-Gefüge anhand des Bildungsbauprozesses (siehe Abb. 3) entwickelt, das ein Zusammenwirken von Handlung, Struktur und Deutungsmustern darlegt.

- Architektonisches ist im Planungsprozess in allen Phasen und Abläufen integriert und damit institutionalisiert, während für pädagogische Themen die Möglichkeiten der Einflussnahme beschränkt sind (Frage der Ressourcen).

- Deutungsmuster stellen ein vermittelndes Element dar und liegen an der Schnittstelle von Handlung und Struktur (Bögelein \& Vetter, 2019, S. 15). Im Prozessverlauf wird besonderes Gewicht auf finanzielle, zeitliche und räumliche (architektonische) Strukturen gelegt. Pädagogische Deutungsmuster verlieren infolge dessen an Bedeutung und kommen daher in ihrer an sich vermittelnden Funktion nur in geringem Maß zum Tragen.

- Projektstrukturen geben gewisse Abläufe vor und sind bestimmend. Zur Erläuterung: Die Umsetzung eines bewilligten Bauentwurfes erfordert in festgesetzten Besprechungsabläufen vor allem Antworten auf finanzielle, bautechnische und architektonische Fragestellungen. Daraus lässt sich ableiten, warum vor allem nicht-pädagogische Deutungsmuster von Akteur*innen zum Tragen kommen. Zudem betrachten Pädagog*innen ihre Vorstellungen in Verhandlungen zum Teil als weniger bedeutsam oder setzten geringe Erwartungen in die Berücksichtigung ihrer Anliegen. Regeln, Ressourcen, Machtverhältnisse, Projektstrukturen und finanzielle Strukturen tragen zum Zurückdrängen pädagogischer Vorstellungen bei.

- Ein Strukturproblem zeigt sich bei der Transferproblematik zwischen Wissenschaft und Praxis sowie Architektur und Pädagogik im Bildungsbau. Nicht immer sind bereits vorhandenes Wissen und Erkenntnisse aus Studien (Beck \& Bonß, 1989) entscheidend für die Wahl eines Projektes. Dies stellt keine neue Erkenntnis dar, da Studien der Wissenschaftsforschung dieses Phänomen bereits vor über 30 Jahren belegten. Es wird anhand der vorliegenden Arbeit sichtbar gemacht, dass diese Problematik noch nicht überwunden ist. 

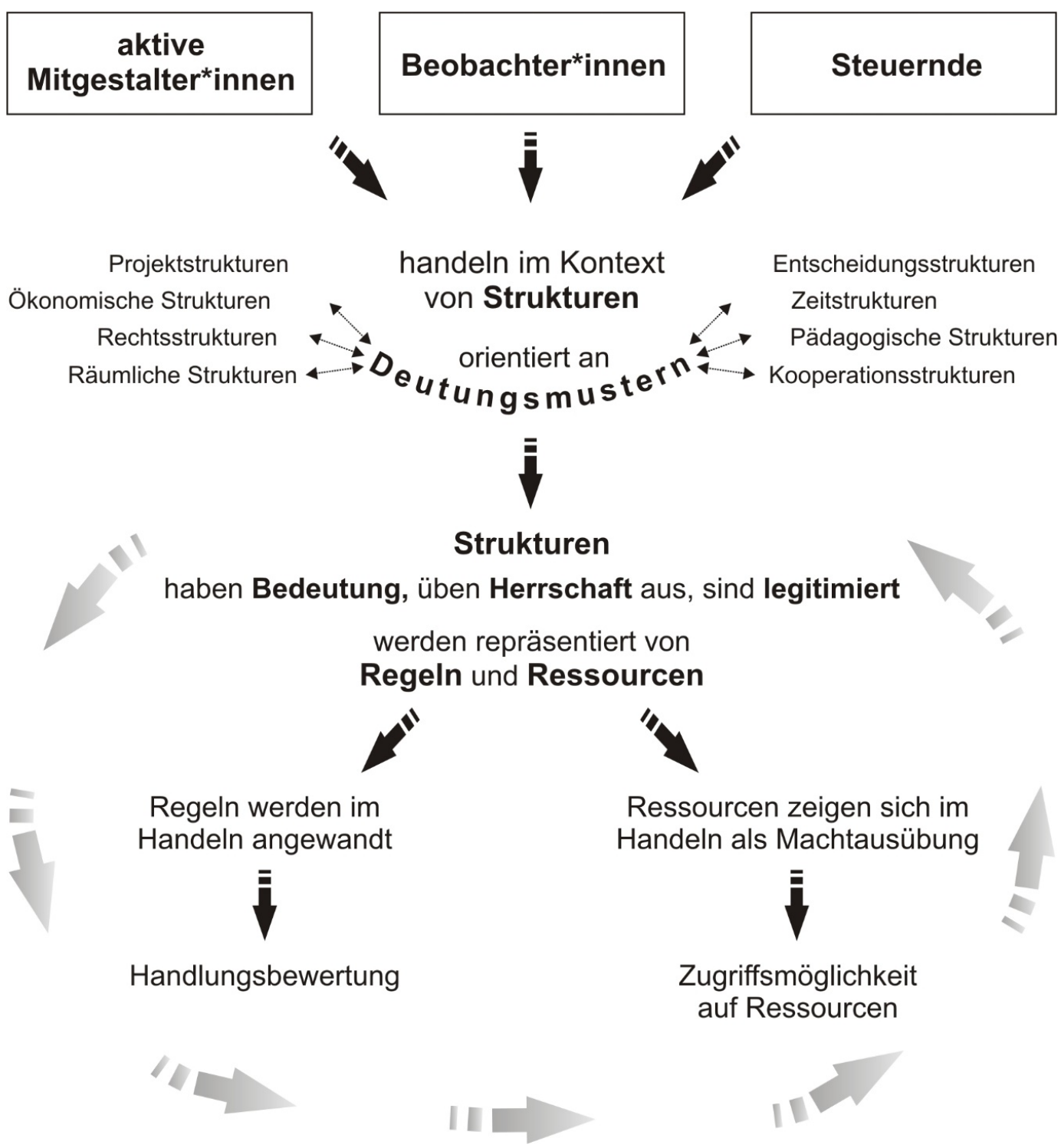

Abbildung 3: Prinzip des Handlungs-Struktur-Gefüges (eigene Darstellung mit Bezug zu Giddens, 1988)

Akteur*innen im Bildungsbau beziehen sich in ihrem Handeln auf Strukturen und werden dabei von ihren Deutungsmustern geleitet. Dabei trifft etwas Starres (Strukturen) auf etwas Aktives (Deutungsmuster), das in Bewegung ist. Das Aufeinandertreffen geschieht in einem rekursiven Prozess, in dem sich beide gegenseitig beeinflussen. Strukturen haben nach Giddens (1988) eine Bedeutung, üben Herrschaft aus und sind legitimiert. Kennzeichnend ist, dass sie von Regeln und Ressourcen repräsentiert werden. Regeln werden im Handeln der Akteur*innen angewandt und führen zu einer Handlungsbewertung, während sich Ressourcen im Handeln als Machtausübung zeigen. Die Zugriffsmöglichkeit auf Ressourcen bzw. deren Mobilisierung entscheidet vielfach über die Möglichkeiten der Einflussnahme.

\section{Schlussfolgerungen}

Schulbau-Empfehlungen und Normen zum Schulhausbau werden wenig eingehalten oder berücksichtigt und pädagogische Deutungsmuster erweisen sich als kaum relevant. Damit wird ein Transferproblem von Wissen zwischen Wissenschaft und Praxis angesprochen, wozu die Wissenschaftstransferforschung Erklärungen liefert (Beck \& Bonß, 1989; Oestreicher, 2014). Die Studie führt aus, warum nicht davon ausgegangen werden kann, dass Forschungen zu gutem Schulbau in der Umsetzung von Bildungsbauprojekten ihren Niederschlag finden, und warum pädagogische Vorstellungen zu wenig Beachtung erhalten. Die Verknüpfung der Analysen von 
Projektstrukturen und Deutungsmustern und das Handlungs-Struktur-Gefüge zeigen, dass bestimmte Akteursgruppen (z. B. Architekt*innen, Behördenvertreter*innen) Zugriff auf wirkmächtige Strukturen und damit auf Ressourcen haben, Pädagog*innen jedoch nur eingeschränkt darüber verfügen. Der Fokus dieser Studie kann für den Bildungshausbau Grundlage sein, um ein Umdenken in Planungsabläufen bei Bildungsbauprozessen anzustoßen. Die Ergebnisse können in Studien an der Schnittstelle von Architektursoziologie (Fischer, 2012; Kemnitz, 2003; Lingg, 2016; Reiterer 2017; Schäfers, 2014; Tosch, 2003), Architekturforschung und Schulforschung eingeordnet werden.

\section{Literatur}

Arnold, R. (1985): Deutungsmuster und pädagogisches Handeln in der Erwachsenenbildung. Aspekte einer Sozialpsychologie der Erwachsenenbildung und einer erwachsenenpädagogischen Handlungstheorie. Bad Heilbrunn/Obb.: Klinkhardt (= ein Bd. der Reihe Theorie und Praxis der Erwachsenenbildung).

Beck, U., \& Bonß, W. (Hrsg.) (1989): Weder Sozialtechnologie noch Aufklärung? Analysen zur Verwendung sozialwissenschaftlichen Wissens. Frankfurt/Main: Suhrkamp.

Bögelein, N., \& Vetter, N. (2019): Deutungsmuster als Forschungsinstrument. Grundlegende Perspektiven. In N. Bögelein \& N. Vetter: Der Deutungsmusteransatz. Einführung - Erkenntnisse - Perspektiven (S. 12-38). Weinheim: Beltz Juventa.

Bohnsack, R., Nentwig-Gesemann, I., \& Nohl, A.-M. (Hrsg.) (2013): Die dokumentarische Methode und ihre Forschungspraxis. Grundlagen qualitativer Sozialforschung (3. Auflage). Wiesbaden: Springer.

Bohnsack, R., Geimer, A., \& Meuser, M. (Hrsg.) (2018): Hauptbegriffe qualitativer Sozialforschung (4. vollständig überarbeitete und erweiterte Auflage). Opladen \& Toronto: Barbara Budrich.

Braum, M., \& Hamm, O. (Hrsg.) (2010): Worauf baut die Bildung? Fakten, Positionen, Beispiele.- Basel: Birkhäuser (= Bd. 1 der Reihe Bericht der Baukultur).

Burgdorff, F., \& Imhäuser, K.-H. (2012): Zukunftsfähiger Schulbau. Von der Herausforderung zur Umsetzung. In H. Schröteler-von Brandt, T. Coelen, A. Zeising, \& A. Ziesche (Hrsg.): Raum für Bildung. Ästhetik und Architektur von Lern- und Lebensorten (S. 233-239). Bielefeld: transcript.

Dewe, B. (1984): Kultursoziologische Bildungsforschung. Zum Wechselverhältnis von lebenspraktischen Deutungsmustern und wissenschaftlicher Orientierung. Zeitschrift für Sozialisationsforschung und Erziehungssoziologie, Heft 4, 307-329.

Dewe, B., \& Ferchhoff, W. (1991): Deutungsmuster. In H. Kerber, \& A. Schmieder (Hrsg.): Handbuch Soziologie. Zur Theorie und Praxis sozialer Beziehungen (S. 76-81). Reinbek bei Hamburg: Rowohlt Taschenbuch.

die Baupiloten (2016): Die Baupiloten-Methode. Verfügbar unter: http://www.baupiloten.com/baupilotenmethode/ [18.05.2021].

Fischer, J. (2012): Rekonstruktivismus als soziale Bewegung. Die revolutionäre Rückkehr der okzidentalen Stadt. Zeitschrift für Literatur und Diskussion: Architektur und Stadt,14, Heft 1, 27-42.

Gaus-Hegner, E. (Hrsg.) (2009): Raum erfahren - Raum gestalten: Architektur mit Kindern und Jugendlichen. Oberhausen: Athena.

Giddens, A. (1984): The Constitution of Society. Outline of the Theory of Structuration. Cambridge: Politiy Press.

Giddens, A. (1988): Die Konstitution der Gesellschaft. Grundzüge einer Theorie der Strukturierung. Frankfurt/New York: Campus.

Grossmann, K. (2007): Am Ende des Wachstumsparadigmas? Zum Wandel von Deutungsmustern in der Stadtentwicklung. Der Fall Chemnitz. Bielefeld: transcript.

Hamm, O. (2010): Vorbildlich - Beispiele für Bildungsbauten. In M. Braum, \& O. Hamm (Hrsg.): Worauf baut die Bildung? Fakten, Positionen, Beispiele (S. 52-113). Basel: Birkhäuser (= Bd. 1 der Reihe Bericht der Baukultur).

Hofmann, S. (2013): Entdeckendes Lernen - pädagogische Architektur. In J. Kahlert, J., K. Nitsche, \& K. Zierer (Hrsg.): Räume zum Lernen und Lehren. Perspektiven einer zeitgemäßen Schulraumgestaltung (S. 279284). Bad Heilbrunn: Klinkhardt. 
Hübner, P. (2016): Lernlandschaften entwerfen. In U. Stadler-Altmann (Hrsg.): Lernumgebungen. Erziehungswissenschaftliche und architekturkritische Perspektiven auf Schulgebäude und Klassenzimmer (S. 113-124). Opladen, Berlin \& Toronto: Barbara Budrich.

Jäger-Klein, C., \& Plakolm-Forsthuber, S. (Hrsg.) (2012): Schulbau in Österreich 1996-2011. Wege in die Zukunft. Wien - Graz: Neuer Wissenschaftlicher Verlag.

Kemnitz, H. (2003): „Neuzeitlicher Schulbau“ für eine „moderne Pädagogik“ - Das Beispiel der Berliner Dammwegschule. In F.-J. Jelich, \& H. Kemnitz (Hrsg.): Die pädagogische Gestaltung des Raums. Geschichte und Modernität (S. 249-267). Bad Heilbrunn: Klinkhardt.

Kleemann, F. Krähnke, U., \& Matuschek, I. (2013): Interpretative Sozialforschung. Eine Einführung in die Praxis des Interpretierens (2. korrigierte und aktualisierte Auflage). Wiesbaden: Springer VS.

Lamnek, S., \& Krell, C. (2016): Qualitative Sozialforschung (6. überarbeitete Auflage). Weinheim, Basel: Beltz, (E-Book).

Linder, W. (2015): Voraussetzung gelingender Partizipation - Rezeptbücher für Planerinnen und Planer? In J. Krebühl (Hrsg.): „Es gibt keine Alternative...?!“. Planung heute. Denkanstöße 12 (S. 44-47). Mainz: Stiftung Natur und Umwelt Rheinland-Pfalz.

Lingg, E. (2016): Hochschulbauten im Spannungsfeld von Bildungspolitik und Stadtentwicklung. Wiesbaden: Springer VS (= Bd. 17 der Schriftenreihe Sozialraumforschung und Sozialraumarbeit).

Matthes, J., \& Schütze, F. (1981): Zur Einführung: Alltagswissen, Interaktion und gesellschaftliche Wirklichkeit. In Arbeitsgruppe Bielefelder Soziologen (Hrsg.): Alltagswissen, Interaktion und gesellschaftliche Wirklichkeit (5. Auflage). Opladen: Westdeutscher Verlag (= Bd. 54/55 WV Studium Sozialwissenschaft).

Meuser, N. (Hrsg.) (2014): Handbuch und Planungshilfe Schulbauten. Berlin: DOM publishers.

Montag Stiftung, Urbane Räume, \& Montag Stiftung, Jugend und Gesellschaft (Hrsg.) (2012): Schulen planen und bauen. Grundlagen und Prozesse (2. Auflage). Berlin: Jovis.

Montag Stiftung, Urbane Räume, Montag Stiftung, Jugend und Gesellschaft, Bund deutscher Architekten, \& Verband Bildung und Erziehung (Hrsg.) (2013): Leitlinien für leistungsfähige Schulbauten in Deutschland. Bonn, Berlin: labor b designbüro.

Neuberger, O. (1995): Mikropolitik. Der alltägliche Aufbau und Einsatz von Macht in Organisationen. Stuttgart: Ferdinand Enke.

Nohl, A.-M. (2013): Komparative Analyse. Forschungspraxis und Methodologie dokumentarischer Interpretation. In R. Bohnsack, I. Nentwig-Gesemann, \& A.-M. Nohl (Hrsg.): Die dokumentarische Methode und ihre Forschungspraxis. Grundlagen qualitativer Sozialforschung (S. 271-293) (3. Auflage). Wiesbaden: Springer.

Nohl, A.-M. (2017): Interview und dokumentarische Methode. Anleitungen für die Forschungspraxis (5. aktualisierte und erweiterte Auflage). Wiesbaden: Springer VS.

Oestreicher, E. (2014): Wissenstransfer in Professionen. Grundlagen, Bedingungen und Optionen. Opladen, Berlin \& Toronto: Budrich UniPress.

Oevermann, U. (2001): Zur Analyse der Struktur von sozialen Deutungsmustern. In S. Kutzner, C. Magnin, C. Scheid, M. Silkenbeumer, \& A. Wernet (Hrsg.): Sozialer Sinn (S. 3-33). (Original 1973, unveröffentlichtes Manuskript).

Oevermann, U. (2001): Die Struktur sozialer Deutungsmuster - Versuch einer Aktualisierung. In S. Kutzner, C. Magnin, C. Scheid, M. Silkenbeumer, \& A. Wernet (Hrsg.): Sozialer Sinn (S. 35-82).

Pampe, B. (2016): Pädagogische Architektur 2016. Verfügbar unter: http://schulen-planen-undbauen.de/2016/01/26/paedagogische-architektur-2016 [18.05.2021].

Plattform SchulUMbau (2010): Charta für die Gestaltung von Bildungseinrichtungen des 21. Jahrhunderts. Verfügbar unter: http://www.aap.or.at/beratung/Charta_Schulumbau.pdf [18.05.2021].

Prechter, G. (2013): Architektur als soziale Praxis. Akteure zeitgenössischer Baukulturen: Das Beispiel Vorarlberg. Wien: Böhlau.

Reiterer, S. (2017): Architektur in der kulturellen Bildung. In G. Weiß (Hrsg.): Kulturelle Bildung - Bildende Kultur. Schnittmengen von Bildung, Architektur und Kunst (S. 131-139). Bielefeld: transcript. 
Schäfers, B. (2014): Architektursoziologie. Grundlagen - Epochen - Themen (3. aktualisierte und erweiterte Auflage). Wiesbaden: Springer VS.

Schopper, M. (2021): Pädagogik und Architektur im Schulhausbau. Eine Fallstudie zu pädagogischen Vorstellungen von Beteiligten an Bildungsbauprozessen. Publikationsserver der Universität Potsdam: https://doi.org/10.25932/publishup-50318, https://nbn-resolving.org/urn:nbn:de:kobv:517-opus4503181.

Schwarz, M. (2017): Ökosponsoring als Kommunikationsinstrument an der Schnittstelle ethischer und politischer Problemstellungen. In K. Berr (Hrsg.): Architektur und Planungsethik. Zugänge, Perspektiven, Standpunkte (S. 71-83). Wiesbaden: Springer.

Sprecher Mathieu, F. (2010): Moderne Schulanlagen. Umweltgerechte Bauplanung für eine neue Lernkultur. Zürich: vdf.

Sydow, J., \& Wirth, C. (Hrsg.) (2014): Organisation und Strukturation. Eine fallbasierte Einführung. Wiesbaden: Springer VS (= Bd. der Schriftenreihe Organisationssoziologie).

Tosch, F. (2003): Spiegelungen pädagogischer Reformen am Schulbau der Weimarer Republik. In F.-J. Jelich, \& H. Kemnitz (Hrsg.): Die pädagogische Gestaltung des Raums. Geschichte und Modernität (S. 231-247). Bad Heilbrunn: Klinkhardt.

von Zadow, A. (2009): Stadt im Umbau. Neue urbane Horizonte. In SIR - Salzburger Institut für Raumordnung \& Wohnen (Hrsg.): Stadt im Umbau - neue urbane Horizonte. Tagungsband zur UN-Dekade „Bildung und nachhaltige Entwicklung" 2005-2014 (S. 16-22). Salzburg.

Walden, R., \& Borrelbach, S. (2014): Schulen der Zukunft (8. Auflage). Kröning: Asanger.

Watschinger, J., \& Kühebacher J. (Hrsg.) (2007): Schularchitektur und neue Lernkultur. Neues Lernen - neue Räume. Ein Projekt des Pädagogischen Instituts für die Deutsche Sprachgruppe, Bozen. Bern: hep. 